\title{
Article
}

\section{The early infrared temporal development of Nova Delphini 2013 (v339 del) observed with the stratospheric observatory for infrared astronomy (Sofia) and from the ground}

Gehrz, RD, Evans, A, Helton, LA, Shenoy, DP, Banerjee, DPK, Woodward, CE, Vacca, WD, Dykhoff, DA, Ashok, NM, Cass, AC, Carlon, RL, Corgan, DT, Eyres, SPS, Joshi, V, Keller, LD, Krautter, J, Liimets, T, Rushton, $M$ and Starrfield, S

Available at http://clok.uclan.ac.uk/15822/

Gehrz, RD, Evans, A, Helton, LA, Shenoy, DP, Banerjee, DPK, Woodward, CE, Vacca, WD, Dykhoff, DA, Ashok, NM et al (2015) The early infrared temporal development of Nova Delphini 2013 (v339 del) observed with the stratospheric observatory for infrared astronomy (Sofia) and from the ground. The Astrophysical Journal, 812 (2). p. 132. ISSN 1538-4357

It is advisable to refer to the publisher's version if you intend to cite from the work. http://dx.doi.org/10.1088/0004-637X/812/2/132

For more information about UCLan's research in this area go to http://www.uclan.ac.uk/researchgroups/ and search for <name of research Group>.

For information about Research generally at UCLan please go to http://www.uclan.ac.uk/research/

All outputs in CLoK are protected by Intellectual Property Rights law, including Copyright law. Copyright, IPR and Moral Rights for the works on this site are retained by the individual authors and/or other copyright owners. Terms and conditions for use of this material are defined in the policies page. 


\title{
THE EARLY INFRARED TEMPORAL DEVELOPMENT OF NOVA DELPHINI 2013 (V339 DEL) OBSERVED WITH THE STRATOSPHERIC OBSERVATORY FOR INFRARED ASTRONOMY (SOFIA) AND FROM THE GROUND
}

\author{
R. D. Gehrz ${ }^{1}$, A. Evans $^{2}$, L. A. Helton ${ }^{3}$, D. P. Shenoy ${ }^{1}$, D. P. K. Banerjez ${ }^{4}$, C. E. Woodward ${ }^{1}$, W. D. Vacca ${ }^{3}$,

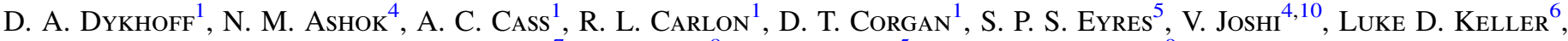 \\ J. Krautter ${ }^{7}$, T. Limmets ${ }^{8}$, M. Rushton ${ }^{5}$, and S. Starrfield 9 \\ ${ }^{1}$ Minnesota Institute for Astrophysics, School of Physics and Astronomy, 116 Church Street, S. E., \\ University of Minnesota, Minneapolis, Minnesota 55455, USA; gehrz@astro.umn.edu \\ 2 Astrophysics Group, Keele University, Keele, Staffordshire, ST5 5BG, UK \\ ${ }^{3}$ USRA-SOFIA Science Center, NASA Ames Research Center, Moffett Field, CA 94035, USA \\ ${ }^{4}$ Astronomy and Astrophysics Division, Physical Research Laboratory, Ahmedabad 380009, India \\ ${ }^{5}$ Jeremiah Horrocks Institute, University of Central Lancashire, Preston PR1 2HE, UK \\ ${ }^{6}$ Department of Physics and Astronomy, 264 Center for Natural Sciences, Ithaca College, Ithaca, NY 14850, USA \\ ${ }^{7}$ Landessternwarte-Zentrum für Astronomie der Universität, Königstuhl, D-69117 Heidelberg, Germany \\ ${ }^{8}$ Tartu Observatory, 61602 Tõravere, Estonia; Institute of Physics, University of Tartu, Ravila 14c, 50411, Tartu, Estonia \\ ${ }^{9}$ School of Earth and Space Exploration, Arizona State University, Box 871404, Tempe, AZ 85287-1404, USA \\ Received 2015 May 8; accepted 2015 September 14; published 2015 October 16
}

\begin{abstract}
We present ground-based infrared photometry, $J H K$ spectroscopy, and 5-28 $\mu \mathrm{m}$ SOFIA FORCAST spectroscopy documenting the early temporal development of Nova Delphini 2013 (V339 Del). We derive a distance of $\sim 4.5 \mathrm{kpc}$ using data available from the early expansion of the fireball. This distance gives an outburst luminosity of $\sim 8.3 \times 10^{5} L_{\odot}$ making V339 Del the most luminous CO nova on record. Our data provide new constraints on the ejected gas mass and the dust yield in fast $\mathrm{CO}$ novae. The ejected gas mass as estimated by the cutoff wavelength during the free-free emission phase is $\sim 7.5 \times 10^{-5} M_{\odot}$. There is evidence for the formation of $\sim 1.2$ $( \pm 0.4) \times 10^{-7} M_{\odot}$ of dust about 102 days after outburst. The gas to dust ratio of $\sim 470 / 1-940 / 1$ implies that dust production was much less efficient in V339 Del than is the case for most CO novae.
\end{abstract}

Key words: circumstellar matter - infrared: stars - novae, cataclysmic variables - stars: individual (V339 Del) stars: winds, outflows

\section{INTRODUCTION}

Classical novae $(\mathrm{CNe})$, explosions resulting from thermonuclear runaways (TNRs) on the surfaces of white dwarf (WD) stars in close binary systems, inject gas and dust into the interstellar medium (ISM). Our group has engaged in a forty year program of infrared (IR) observations of the temporal development of $\mathrm{CN}$ eruptions to determine important physical parameters that characterize the nova explosion and the extent to which nova ejecta affect ISM abundances on both local and global scales (Gehrz 1988, 1999, 2008; Evans \& Gehrz 2012; Gehrz et al. 2014). Nova explosions on some carbon-oxygen (CO) WDs are known to result in the condensation of dust whose composition includes carbon, silicates, $\mathrm{SiC}$, and hydrocarbons (see Evans \& Rawlings 2008; Gehrz 2008; Evans \& Gehrz 2012 for reviews). The ejecta of nova explosions on more massive oxygen-neon $(\mathrm{ONe})$ WDs are known to have produced ejecta with substantial enrichments of $\mathrm{C}, \mathrm{N}, \mathrm{O}, \mathrm{Ne}, \mathrm{Mg}$, and $\mathrm{Al}$ over solar abundance (e.g., Helton et al. 2012).

Nova Delphini 2013 (PNV J20233073+2046041, V339 Del) was discovered by K. Itagaki on 2013 August 14.584 UT (JD 2456519.084) at a visual magnitude of $\sim+6.8$ (Nakano et al. 2013). During the next few days, it brightened steadily until it reached a peak visual brightness of $m_{\mathrm{v}}=+4.4$ on 2456521.4 , making it one of the brightest novae in almost 20 years. V339 Del declined rapidly in visual intensity thereafter, as shown in the light curve plotted in Figure 1. The early light

\footnotetext{
${ }^{10}$ Present address: Inter-University Centre for Astronomy and Astrophysics, Post Bag 4, Pune University Campus, Ganeshkhind, Pune 411007, India.
}

decline rate $\left(\sim 0.2\right.$ mag day $\left.^{-1}\right)$ and peak brightness suggested that V339 Del might be an ONe nova similar in behavior to QU Vul (Nova Vul 1984\#2; Gehrz et al. 1985) or V838 Her (Nova Her 1991; Woodward et al. 1992). We activated Target of Opportunity (ToO) grism observations using the NASA Stratospheric Observatory for IR Astronomy (SOFIA; Young et al. 2012) in anticipation of the possible early formation of strong IR metallic forbidden lines such as [Ne II] at $12.8 \mu \mathrm{m}$. However, evidence for a very weak dust formation event in the observed spectral energy distribution (SED) after $\sim 1.5$ months (Cass et al. 2013; Shenavrin et al. 2013) suggests that V339 Del is more likely a fast CO nova similar to V1668 Cyg (Nova Cyg 1978; Gehrz et al. 1980b) or Nova LMC 1991 (Schwarz et al. 2001).

We monitored V339 Del at optical and IR wavelengths over the first three months of its evolution. Here we present results of IR photometry at O'Brien Observatory and Mount Abu, spectroscopic observations with the FORCAST instrument aboard SOFIA, and ground-based near-infrared (near-IR) spectroscopy from the Mount Abu Observatory that is contemporaneous with the SOFIA spectrum.

\section{OBSERVATIONS}

\subsection{Thermal IR Spectral Observations with SOFIA}

Mid-IR spectroscopic observations of V339 Del were obtained using the Faint Object IR CAmera for the SOFIA Telescope (FORCAST; Herter et al. 2012) mounted at the Nasmyth focus of the $2.5 \mathrm{~m}$ telescope of the newly 


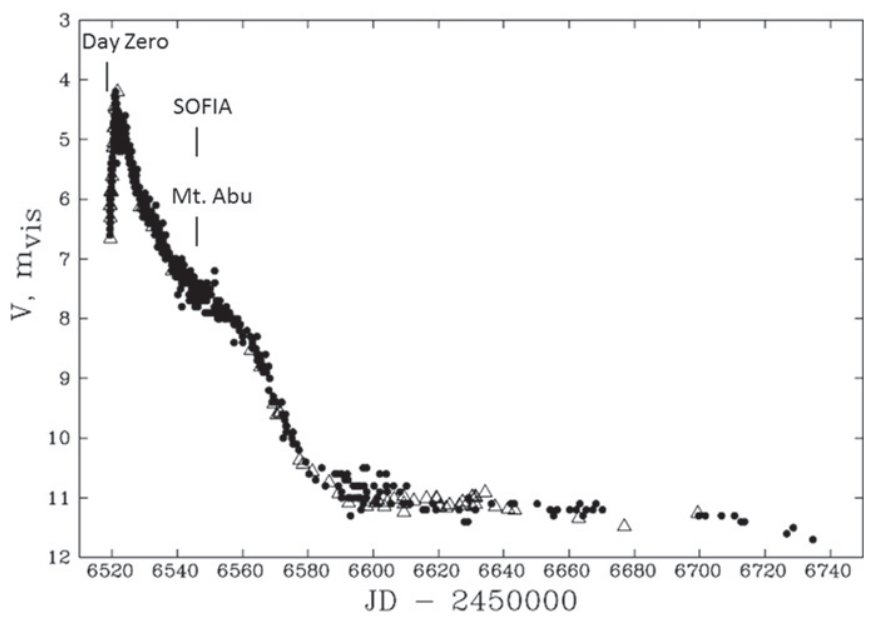

Figure 1. Visual light curve, courtesy of the British Astronomical Association and The Astronomer magazine. Solid circles are visual estimates. Open triangles are data obtained using a charge coupled device (CCD) with a V filter. Day Zero, and the dates of the contemporaneous SOFIA and Mt. Abu spectral data are indicated by vertical ticks.

commissioned SOFIA Observatory. The FORCAST instrument is a dual-channel MIR imager and grism spectrometer operating from 5 to $40 \mu \mathrm{m}$. Light is fed to two $256 \times 256$ pixel blocked-impurity-band (BIB) arrays, each with a plate scale of 0 ! 768 pixel $^{-1}$ and a distortion corrected field of view of 3 !" $2 \times 3$ !" 4. The Short Wavelength Camera (SWC) covers the spectral region from 5 to $25 \mu \mathrm{m}$, while the Long Wavelength Camera (LWC) operates at wavelengths from 25 to $40 \mu \mathrm{m}$. Observations of novae with SOFIA have been long anticipated because a large number of IR forbidden lines of metals, many of them unobservable from the ground, fall within the bandpasses of the FORCAST grisms (see Table 1). These lines are quenched at high densities, but can dominate the IR spectrum once electron densities have fallen below $n_{\mathrm{e}} \sim 10^{6}-10^{7} \mathrm{~cm}^{-3}$ in the expanding ejecta.

The data were acquired during a single flight (F128; originating from Palmdale, CA) at an altitude of $39,000 \mathrm{ft}$, $(11.89 \mathrm{~km})$ on 2013 September 10.3 UT (JD 2456545.8; day 27.4) as part of our SOFIA Cycle 1 ToO program to observe CNe in outburst (P.I. Gehrz, proposal ID 01_0017). V339 Del was imaged in the SWC using the F064 filter $\left(\lambda_{\text {eff }}=6.4 \mu \mathrm{m}\right.$; $\Delta \lambda=0.14 \mu \mathrm{m})$ to position the target on the slit. Spectroscopic data were obtained using three grisms, two in the SWC (G063 and G111) using a narrow long-slit $\left(2\right.$ ". $\left.4 \times 191^{\prime \prime}\right)$ yielding a spectral resolution of $R=\lambda / \Delta \lambda \sim 140-300$, and one in the LWC (G227) using a $4.7 \times 191^{\prime \prime}$ slit yielding a spectral resolution of $R \sim 70$. Both imaging and spectroscopy were conducted using a 2-point chop/nod in the default Nod-MatchChop (NMC) mode with a $60^{\prime \prime}$ chop/nod amplitude at angles of $30^{\circ} / 210^{\circ}$ in the science instrument reference frame. Details of the operating modes of FORCAST on SOFIA have been described by Herter et al. (2012) and Temi et al. (2014). The negative beams were not on the slit. Details of the SOFIA FORCAST observations of V339 Del are provided in Table 2.

The data were reduced using the SOFIA facility pipeline package, Redux (Clarke et al. 2015). The FORCAST Data Pipeline performs a number of corrections on the 2D data for both imaging and spectroscopy modes, including cleaning bad pixels, droop correction, nonlinearity correction, flat fielding, background subtraction, and jail-bar correction (see Edwards et al. 2010 for detailed descriptions of some of these effects). In the case of imaging data, Redux performs a distortion correction and merges and registers the files. Redux also incorporates the fspextool software package, which is a modification of the Spextool package (Cushing et al. 2004) developed for the SpeX NIR spectrograph at the NASA IR Telescope Facility (Rayner et al. 2003). These routines perform the coaddition of imaging data and the extraction and combination of the grism data.

Aperture photometry was performed on the pipeline processed $6.3 \mu \mathrm{m}$ acquisition images according to the same parameters used for extraction of the flux from the calibrators, namely with an aperture radius of 12 pixels (which corresponds to $\sim 9$ !" 43 at the FORCAST platescale of 0 ". 786 pixel $^{-1}$ ), an inner radius of the sky annulus of 15 pixels, and an annular width of 10 pixels. Sky subtraction was applied using the median of the background annulus. The source was point-like $(\mathrm{FWHM}=4.15$ pixel $=3$ !' 2$)$, consistent with the resolution typically achieved with FORCAST using the $6.4 \mu \mathrm{m}$ F064 filter. The calibration factor applied to the flux measurement was included in the Level 3 data distribution and was derived from 17 calibrator observations of $\beta$ And, $\alpha$ Boo, and $\alpha$ Cet in a manner following that presented by Herter et al. (2013). The FORCAST spectra are shown in Figures 2 and 4 with the $6.34 \mu \mathrm{m}$ photometric data point shown overlaid in red in Figure 2. We calculate a flux for V339 Del of $F_{\mathrm{v}}=3.58 \pm$ $0.51 \mathrm{Jy}$ at $6.34 \mu \mathrm{m}$. This value was derived assuming a "flatspectrum" source. Color corrections ${ }^{11}$ for these observations are negligible for any reasonable power law spectral energy distribution $\left(F_{\mathrm{v}} \propto v^{\alpha}\right)$ where $-3<\alpha<3 ; \alpha \sim-1$ for the Day 27 V339 Del FORCAST spectrum (see Figure 4). It is evident that the spectrum is dominated by hydrogen emission and that no metallic forbidden lines are present. No forbidden lines were necessarily expected at this early epoch because of the high shell density (see Section 3 below). Unfortunately, circumstances prevented us from observing the nova with SOFIA at a later epoch when shell density would have been low enough for metallic forbidden lines to have been present.

Spectroscopic analysis was performed on the Level 3, pipeline calibrated data (basic calibrated data; BCD). The instrumental errors were dominated by the overall bias level uncertainty in the calibrator, not by the pixel to pixel deviation of the spectra. For this reason, line fitting was performed using multiple techniques, the output of which were used in calculating the mean flux and associated errors as presented in Table 3. The fitting routines included two different Gaussian fitting algorithms, a Gauss error function (erf) profile fit, and direct integration. The flux measurements in Table 3 are the unweighted mean of the individual fit values and the errors are the standard deviation of the mean.

\subsection{IR Photometry at O'Brien Observatory}

We obtained $0.6-11.3 \mu \mathrm{m}$ IR photometric observations of V339 Del on 13 occasions during 2013 August-November using a multi-filter As:Si bolometer mounted on the University of Minnesota's O'Brien Observatory (OBO) $0.76 \mathrm{~m}$ telescope. The standard ABBA beam switching technique was used to cancel the thermal background from the sky and telescope using a $27^{\prime \prime}$ diameter aperture and a throw of $50^{\prime \prime}$ between the

\footnotetext{
11 http://www.sofia.usra.edu/Science/DataProducts/

FORCAST_ColorCorrecns_OC1.pdf
} 
Table 1

Selected Infrared Forbidden Lines within the FORCAST Grism Passbands

\begin{tabular}{|c|c|c|c|c|c|c|c|}
\hline SPECIES & $\lambda(\mu \mathrm{m})$ & SPECIES & $\lambda(\mu \mathrm{m})$ & SPECIES & $\overline{\lambda(\mu \mathrm{m})}$ & SPECIES & $\overline{\lambda(\mu \mathrm{m})}$ \\
\hline [O IV] & 25.91 & [Na VIII] & 6.23 & {$[\mathrm{Al} \mathrm{x}]$} & 6.06 & {$[\mathrm{Mg} \mathrm{v}]$} & 13.54 \\
\hline$[\mathrm{O} v]$ & 32.61 & [Na III] & 7.32 & [Al vI] & 9.12 & [Si VII] & 6.51 \\
\hline$[\mathrm{Ne} \mathrm{VI}]$ & 7.64 & {$[\mathrm{Na}$ VI] } & 8.61 & [Al VII] & 37.6 & [Si VIII] & 18.45 \\
\hline$[\mathrm{Ne}$ VII] & 22 & [Na VIII] & 13.66 & {$[\mathrm{Mg}$ v] } & 5.6 & [S IV] & 10.51 \\
\hline$[\mathrm{Ne} \mathrm{v}]$ & 24.28 & {$[\mathrm{Na}$ IV] } & 21.29 & [Mg IX] & 8.87 & {$[\mathrm{~S} v]$} & 27.1 \\
\hline$[\mathrm{Ne}$ III] & 36.02 & [Al VIII] & 5.85 & [Mg VII] & 9.03 & [S III] & 33.47 \\
\hline
\end{tabular}

Table 2

Parameters of the SOFIA Grism Observations

\begin{tabular}{|c|c|c|c|c|c|c|c|c|}
\hline Channel & Config & Spectral Element & Bandpass $(\mu \mathrm{m})$ & Slit & $R(\lambda / \Delta \lambda)$ & Coadds & Total Int. Time (s) & Zenith Angle start \\
\hline SWC & Spect & G063 & $4.9-8.0$ & LS24 & 180 & 6 & 44.94 & 39.2 \\
\hline SWC & Spect & G111 & $8.4-13.7$ & LS24 & 300 & 6 & 153 & 41.2 \\
\hline
\end{tabular}

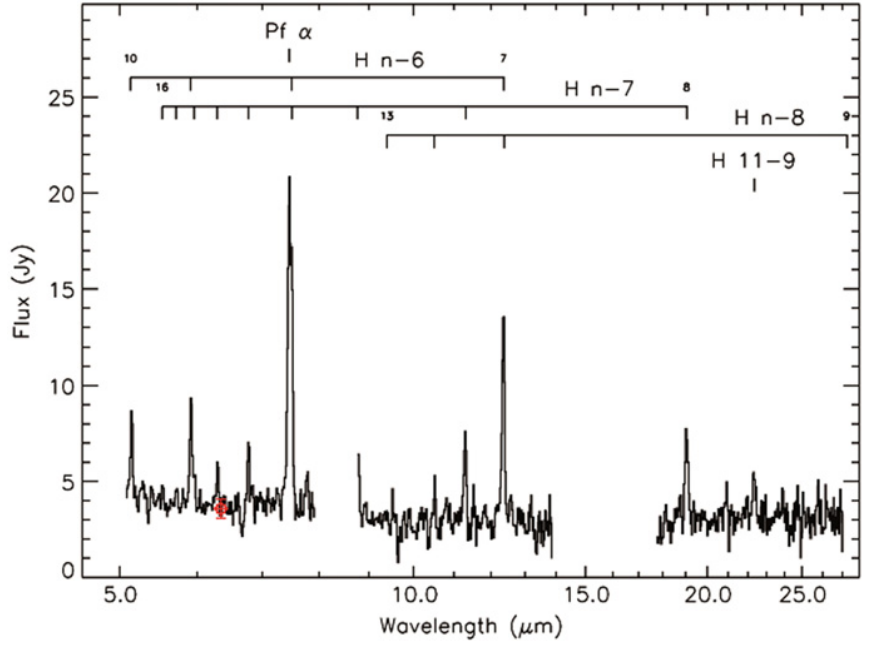

Figure 2. 5-27 $\mu$ m SOFIA FORCAST spectrum obtained on 2013 September 10.3 UT (JD 2456545.8; day 27.4) is dominated by hydrogen recombination lines. We show the expected wavelengths of the relevant hydrogen line sequences above the spectrum. The hydrogen emission is quenched because of the high shell density $\left(n_{\mathrm{e}} \sim 10^{13} \mathrm{~cm}^{-3}\right)$ during this phase of the expansion of the ejecta. No forbidden emission lines of metals are expected at this high a density, and none are observed. The $6.34 \mu \mathrm{m}$ SOFIA photometric data point is shown overlaid in red.

source and reference beams. The photometric system, magnitude scale, method of extinction correction, and absolute flux calibration for the OBO bolometer are given by Gehrz \& Ney (1992) and Gehrz (1997). Arcturus ( $\alpha$ Boo) and Scheat ( $\beta$ Peg) were used to calibrate the observations. The observed magnitudes for V339 Del from OBO and those from NIR observations at Mt. Abu (see Section 2.3 below) are summarized in Table 4 and the NIR light curves are shown in Figure 5. SEDs for some of the important phases in the early development are displayed in Figure 6.

\subsection{Near-IR Photometry and Spectroscopy at Mt. Abu}

Near-IR spectroscopic and photometric observations of V339 Del were carried out using the $1.2 \mathrm{~m}$ telescope of the Mount Abu IR Observatory with the NICMOS3 NIR imager/ spectrograph which uses a $256 \times 256 \mathrm{HgCdTe}$ array (Banerjee
\& Ashok 2012; Banerjee et al. 2013a, 2013b). Photometric observations of the nova were carried out using the NICMOS3 array in the imaging mode. Several frames were obtained in four to five dithered positions, typically offset by $\sim 30^{\prime \prime}$ from each other. Sky frames were generated by median combining the average of each set of dithered frames and these were subsequently subtracted from the nova frames. A nearby fieldstar SAO 88671, observed at the same air mass as the nova, was used as the standard star for photometric calibration. JHK magnitudes of V339 Del were estimated using aperture photometry. Spectrophotometric reduction and analysis was done using IRAF. ${ }^{12}$ Mt. Abu $J H K$ photometry is included in Table 4 and Figures 5 and 6.

The $1.08-2.35 \mu \mathrm{m}$ spectra at $R \sim 1000$, were wavelength calibrated using $\mathrm{OH}$ sky lines and telluric features that register with the stellar spectra. Telluric features were removed using the spectra of a standard star SAO 88944 (2MASS J20260122 +2034558; spectral type A2 V) which lies very close to the nova. The standard star was always observed at similar airmass as the nova, to ensure that the ratioing process (nova spectrum divided by the standard star spectrum) reliably removed the telluric lines. Before the ratioing process, the hydrogen lines in the spectra of the standard star were carefully removed. The ratioed spectra were then multiplied by a blackbody curve corresponding to the standard star's effective temperature to yield the final spectra. The NIR spectrum for 2013 September 10.66 (JD 2456546.16, day 27.76) is shown in Figure 3. Figure 4 shows the entire $1.08-28 \mu \mathrm{m}$ spectrum as recorded by both Mt. Abu and SOFIA on Day $\sim 27$. In addition to the spectral data presented here, we have 17 more epochs of spectroscopic data from Mt Abu and one spectrum with the $3 \mathrm{~m}$ NASA IRTF telescope taken between 2015 August 18 (4 days past outburst) and June 27. Detailed discussion and modeling of this extended data set is beyond the scope of this paper, and will be treated in a forthcoming manuscript.

\footnotetext{
12 IRAF is distributed by the National Optical Astronomy Observatory, which is operated by the Association of Universities for Research in Astronomy (AURA) under cooperative agreement with the National Science Foundation.
} 
Table 3

Sofia Forcast Line Fits ${ }^{\mathrm{a}}$

\begin{tabular}{|c|c|c|c|c|c|c|c|}
\hline Line ID & $\lambda_{\text {rest }}(\mu \mathrm{m})$ & $\overline{\lambda_{\text {avg }}(\mu \mathrm{m})}$ & $\Delta \lambda(\mu \mathrm{m})$ & Flux $\left(10^{-15} \mathrm{~W} \mathrm{~m}^{-2}\right)$ & $\Delta$ Flux $\left(10^{-15} \mathrm{~W} \mathrm{~m}^{-2}\right)$ & $\overline{I_{\ell} / I_{(8-7)}}$ & $\overline{l_{\ell} / I_{(8-7)}}$ \\
\hline HI (10-6) & 5.129 & 5.139 & 0.001 & 18.2 & 1.34 & 2.663 & 0.232 \\
\hline HI (9-6) & 5.908 & 5.916 & 0.001 & 20.4 & 1.19 & 2.987 & 0.222 \\
\hline HI (13-7) & 6.292 & 6.295 & 0.002 & 5.90 & 0.30 & 0.863 & 0.059 \\
\hline HI (12-7) & 6.772 & 6.771 & 0.001 & 7.85 & 0.19 & 1.148 & 0.060 \\
\hline HI $(6-5)^{\mathrm{b}}$ & 7.46 & 7.468 & 0.007 & 78.1 & $\ldots$ & $\ldots$ & $\ldots$ \\
\hline HI (12-8) & 10.503 & 10.509 & 0.003 & 2.84 & 0.16 & 0.415 & 0.030 \\
\hline HI (9-7) & 11.309 & 11.289 & 0.003 & 9.26 & 0.27 & 1.355 & 0.074 \\
\hline $\mathrm{HI}(7-6)^{\mathrm{c}}$ & 12.37 & 12.359 & 0.003 & 20.4 & 2.37 & $\cdots$ & $\cdots$ \\
\hline HI (8-7) & 19.062 & 19.044 & 0.004 & 6.84 & 0.32 & 1.000 & 0.066 \\
\hline HI (11-9) & 22.340 & 22.324 & 0.004 & 2.67 & 0.11 & 0.391 & 0.024 \\
\hline
\end{tabular}

Notes.

${ }^{\mathrm{a}}$ Excludes the blended line complexes as indicated below.

${ }^{b}$ This line is a blend of HI (6-5) + HI (8-6) + HI (11-7).

c This line is a blend of HI (7-6) + HI (11-8).

\section{DATE OF OUTBURST, DISTANCE, AND LUMINOSITY}

In this section we derive physical parameters that are necessary to characterize the eruption and early development of the ejecta of V339 Del that can be used for comparisons with physical parameters that have characterized the development of other novae.

On the rise to maximum, the observed flux comes from the expansion of the fireball. Assuming that the fireball emits like a blackbody, pre-maximum photometry can be used to determine an effective temperature, and the angular diameter of the emitting blackbody. As this essentially provides an "isophotal" rather than a "metric" angular diameter (Evans et al. 1990) the photometry needs to be dereddened. Munari et al. (2013a) estimate that maximum light was on August 16.4 UT (JD 2456520.9) and give pre-maximum BVRI photometry, our analysis of which we summarize in Table 5 . We have dereddened their photometry using their value of $E(B-V)=0.18$ (Munari et al. 2013b) and the extinction law derived by Rieke \& Lebofsky (1985) to fit a blackbody curve for each observation to obtain a temperature, $T_{\mathrm{BB}}$, and a peak flux, $\left(\lambda F_{\lambda}\right)_{\max }$, for the expanding pseudo-photosphere (see Figure 7). Gallagher \& Ney (1976), Gehrz (1999, 2008), Evans \& Gehrz (2012) have shown that these parameters yield a blackbody angular diameter $\theta_{\mathrm{BB}}$ (milliarcseconds) given by:

$$
\theta_{\mathrm{BB}}=2.02 \times 10^{14} \frac{\left[\left(\lambda F_{\lambda}\right)_{\max }\right]^{1 / 2}}{T_{\mathrm{BB}}^{2}} \text { milliarcsec }
$$

where $\left(\lambda F_{\lambda}\right)_{\max }$ is the blackbody emittance of the Planckian maximum in $\mathrm{W} \mathrm{cm}^{-2}$. A simple linear regression from the data in Table 5 gives the blackbody angular diameter (milliarcseconds) as a function of time as:

$$
\theta_{\mathrm{BB}}=(0.130 \pm 0.027) \mathrm{JD}-2.39 \pm 0.55
$$

(see Figure 7) where "JD" is the Julian Day expressed as $2,456,5 \mathrm{XX}-2,456,500$. From Equation (2), we infer that day zero, the date on which the expansion began, was JD $2,456,518.4 \pm 0.5$ (2013 August 13.9 UT). The distance $D$ to the nova can be estimated using the relationship derived by Gehrz (1999) and Evans \& Gehrz (2012):

$$
D=\frac{1.1572 \times 10^{-3} V_{\mathrm{o}}}{d \theta / d t} \mathrm{kpc},
$$

where $d \theta / d t$ is the expansion rate of the diameter in milliarcseconds day ${ }^{-1}$ and $V_{\mathrm{o}}$ is the expansion velocity in $\mathrm{km} \mathrm{s}^{-1}$. We derive an outflow velocity of $505 \mathrm{~km} \mathrm{~s}^{-1}$ by taking half the full line widths at half maximum intensity in a Mt. Abu spectrum obtained on 2013 September 10 UT (see Table 6). A comment is in order concerning the interpretation of the highvelocity wings observed in early epoch optical spectra of V339 Del (Shore et al. 2013b; Skopal et al. 2014) and other CO novae (LW Ser, Prabhu \& Anupama 1987a, 1987b; V1668 Cyg, Kolotilov 1980). Prabhu \& Anupama (1987a, 1987b), Kolotilov (1980), and Schaefer et al. (2014) have all concluded that these high velocities originate in low-density, outlying gas, and that the lower velocity given by the line core is representative of the expansion rate of the bulk of the ejecta. Finally, we note that an examination of Figure 7 in Skopal et al. (2014) shows that the radial velocity deduced from the $\mathrm{H}-\alpha$ wings in V339 Del decreased steadily from $1600 \mathrm{~km} \mathrm{~s}^{-1}$ on 2013 August 15 to about $700 \mathrm{~km} \mathrm{~s}^{-1}$ on 2013 August 20 as the low density outer layers dissipated. Thus, the expansion velocity for the principal ejecta of $505 \mathrm{~km} \mathrm{~s}^{-1}$ deduced from the 2013 September $10 \mathrm{Mt} \mathrm{Abu} \mathrm{spectrum} \mathrm{is} \mathrm{not} \mathrm{at} \mathrm{all}$ unreasonable.

Given a velocity of $505 \mathrm{~km} \mathrm{~s}^{-1}$ for the principal ejecta, the expansion rate given by Equation (2) yields a distance using Equation (3) of $D=4.4953 \pm 0.8 \mathrm{kpc}$. Our numbers are in reasonable agreement with the values of $d \theta$ mas $/ d t=0.156 \pm$ 0.003 mas day $^{-1}$, day zero $=$ JD 2456518.277 (2013 August $13.79)$, and $D=4.54 \pm 0.59 \mathrm{kpc}$ derived by Schaefer et al. (2014) from IR spatial interferometry. We take the distance to V339 Del to be $4.5 \mathrm{kpc}$ and Day zero to be JD $=2456518.4$ in the discussion that follows.

The outburst luminosity, $L_{\mathrm{o}}$, of a nova at a distance of $D$ $(\mathrm{kpc})$ from the Earth is given by the relationship derived by Gehrz (1999, 2008) and Evans \& Gehrz (2012):

$$
L_{\mathrm{o}}\left(L_{\odot}\right)=4.03 \times 10^{17} D^{2}\left[\left(\lambda F_{\lambda}\right)_{\max }\right]
$$

where the value of $\left(\lambda F_{\lambda}\right)_{\max }$ is that measured at maximum light. From the data given in Table 5 , the peak value of $\left(\lambda F_{\lambda}\right)_{\max }$ at maximum light was $10.21 \times 10^{-14} \mathrm{~W} \mathrm{~cm}^{-2}$. Given the distance of $4.5 \mathrm{kpc}$, the peak outburst luminosity of V339 Del yielded by Equation (4) is $L_{\mathrm{o}} \sim 8.3 \times 10^{5} L_{\odot}$, about 13 
Table 4

RIJKLMN Photometry for V339 Del (Magnitudes)

\begin{tabular}{|c|c|c|c|c|c|c|c|c|c|c|c|c|c|c|c|}
\hline YEAR & UT Day & $\begin{array}{c}\text { JD } \\
2450000+\end{array}$ & $\begin{array}{c}\text { Days Since } \\
\text { Outburst }\end{array}$ & $R 0.6 \mu \mathrm{m}$ & $I 0.85 \mu \mathrm{m}$ & $J 1.25 \mu \mathrm{m}$ & $H 1.65 \mu \mathrm{m}$ & $K 2.2 \mu \mathrm{m}$ & $L 3.6 \mu \mathrm{m}$ & $M 4.9 \mu \mathrm{m}$ & $N 10 \mu \mathrm{m}$ & $\begin{array}{c}N^{\prime} 1 \\
10.3 \mu \mathrm{m}\end{array}$ & $\begin{array}{c}N^{\prime} 2 \\
11.6 \mu \mathrm{m}\end{array}$ & REF & SITE \\
\hline 2013 & $\begin{array}{l}\text { AUG } \\
16.20\end{array}$ & 6520.70 & 2.30 & $5.4(0.1)$ & $5.2(0.1)$ & $4.4(0.1)$ & $4.3(0.1)$ & $4.3(0.1)$ & $4.5(.2)$ & $\cdots$ & $\cdots$ & $\cdots$ & $\cdots$ & $\begin{array}{l}\text { Atel } \\
5299\end{array}$ & OBO \\
\hline 2013 & $\begin{array}{l}\text { AUG } \\
17.15\end{array}$ & 6521.65 & 3.25 & $4.7(0.1)$ & $4.5(0.1)$ & $4.3(0.1)$ & $3.9(0.1)$ & $3.9(0.1)$ & $3.9(0.1)$ & $3.5(0.2)$ & $\cdots$ & $\cdots$ & $\cdots$ & $\begin{array}{l}\text { Atel } \\
5299\end{array}$ & OBO \\
\hline 2013 & $\begin{array}{l}\text { AUG } \\
21.17\end{array}$ & 6525.67 & 7.27 & $5.1(0.1)$ & $4.6(0.1)$ & $4.6(0.1)$ & $4.1(0.1)$ & $3.9(0.1)$ & $2.7(0.1)$ & $2.2(0.2)$ & $\cdots$ & $\cdots$ & $\cdots$ & & OBO \\
\hline 2013 & $\begin{array}{l}\text { AUG } \\
23.13\end{array}$ & 6527.63 & 9.23 & $5.1(0.1)$ & $4.9(0.1)$ & $5.1(0.1)$ & $4.0(0.1)$ & $3.7(0.1)$ & $3.1(0.1)$ & $2.7(0.1)$ & $\cdots$ & $\cdots$ & $\cdots$ & & OBO \\
\hline 2013 & $\begin{array}{l}\text { AUG } \\
28.13\end{array}$ & 6532.63 & 14.23 & $5.6(0.1)$ & $5.2(0.1)$ & $4.5(0.1)$ & $4.7(0.1)$ & $4.7(0.1)$ & $3.3(0.1)$ & $1.8(0.4)$ & $\cdots$ & $\cdots$ & $\cdots$ & $\begin{array}{l}\text { Atel } \\
5340\end{array}$ & OBO \\
\hline 2013 & $\begin{array}{l}\text { AUG } \\
31.15\end{array}$ & 6535.65 & 17.25 & $6.9(0.4)$ & $7.4(0.2)$ & $6.7(0.3)$ & $5.4(0.2)$ & $5.4(0.1)$ & $3.8(0.2)$ & $3.4(0.5)$ & $1.2(0.4)$ & $\cdots$ & $\cdots$ & & OBO \\
\hline 2013 & $\begin{array}{l}\text { AUG } \\
31.50\end{array}$ & 6536.00 & 17.60 & $\cdots$ & $\cdots$ & $\cdots$ & $5.6(0.2)$ & $5.4(0.2)$ & $4.4(0.3)$ & $\cdots$ & $\cdots$ & $\cdots$ & $\cdots$ & $\begin{array}{l}\text { Atel } \\
5419\end{array}$ & OBO \\
\hline 2013 & $\begin{array}{c}\text { SEP } \\
03.14\end{array}$ & 6538.64 & 20.24 & $5.7(0.2)$ & $5.5(0.1)$ & $4.4(0.2)$ & $5.8(0.4)$ & $5.6(0.2)$ & $4.4(0.5)$ & $2.2(0.4)$ & $2.1(0.3)$ & $\cdots$ & $\cdots$ & & OBO \\
\hline 2013 & $\begin{array}{c}\text { SEP } \\
07.68\end{array}$ & 6543.18 & 24.78 & $\cdots$ & $\cdots$ & $5.47(0.02)$ & $5.58(0.02)$ & $5.36(0.04)$ & $\cdots$ & $\cdots$ & $\cdots$ & $\cdots$ & $\cdots$ & & $\mathrm{ABU}$ \\
\hline 2013 & $\begin{array}{c}\text { SEP } \\
10.72\end{array}$ & 6546.22 & 27.82 & $\cdots$ & $\cdots$ & $5.64(0.02)$ & $5.71(0.03)$ & $5.54(0.04)$ & $\cdots$ & $\cdots$ & $\cdots$ & $\cdots$ & $\cdots$ & & $\mathrm{ABU}$ \\
\hline 2013 & $\begin{array}{l}\text { SEP } \\
14.77\end{array}$ & 6550.27 & 31.87 & $\cdots$ & $\cdots$ & $5.70(0.01)$ & $5.81(0.02)$ & $5.35(0.07)$ & $\cdots$ & $\cdots$ & $\cdots$ & $\cdots$ & $\cdots$ & & $\mathrm{ABU}$ \\
\hline 2013 & $\begin{array}{c}\text { SEP } \\
17.04\end{array}$ & 6552.54 & 34.14 & $6.0(0.1)$ & $7.0(0.1)$ & $3.6(0.1)$ & $5.5(0.3)$ & $5.3(0.5)$ & $4.5(0.3)$ & $2.1(0.2)$ & $1.8(0.2)$ & $\cdots$ & $\cdots$ & & OBO \\
\hline 2013 & $\begin{array}{c}\text { SEP } \\
20.67\end{array}$ & 6556.17 & 37.77 & $\ldots$ & $\cdots$ & $5.78(0.02)$ & $5.74(0.02)$ & $5.11(0.04)$ & $\cdots$ & $\cdots$ & $\cdots$ & $\cdots$ & $\cdots$ & & $\mathrm{ABU}$ \\
\hline 2013 & $\begin{array}{c}\text { SEP } \\
23.10\end{array}$ & 6558.60 & 40.20 & $6.7(0.4)$ & $6.7(0.5)$ & $4.3(0.3)$ & $5.5(0.7)$ & $4.2(0.1)$ & $3.9(0.4)$ & $\cdots$ & $\cdots$ & $\cdots$ & $\cdots$ & & OBO \\
\hline 2013 & $\begin{array}{c}\text { SEP } \\
25.18\end{array}$ & 6560.68 & 42.28 & $\cdots$ & $5.4(0.2)$ & $\cdots$ & $\cdots$ & $5.0(0.4)$ & $2.6(0.3)$ & $\cdots$ & $\cdots$ & $\cdots$ & $\cdots$ & & $\mathrm{OBO}$ \\
\hline 2013 & $\begin{array}{l}\text { OCT } \\
31.56\end{array}$ & 6597.06 & 78.66 & $\cdots$ & $\cdots$ & $8.32(0.03)$ & $6.67(0.04)$ & $5.15(0.03)$ & $\cdots$ & $\cdots$ & $\cdots$ & $\cdots$ & $\cdots$ & & $\mathrm{ABU}$ \\
\hline 2013 & $\begin{array}{l}\text { NOV } \\
19.10\end{array}$ & 6615.60 & 97.20 & $\cdots$ & $\cdots$ & $\cdots$ & $\cdots$ & $>5.0(3)$ & $>3.1(3)$ & $1.4(0.1)$ & $>0.8$ & $-0.1(0.4)$ & $\cdots$ & & OBO \\
\hline 2013 & $\begin{array}{l}\text { NOV } \\
23.56\end{array}$ & 6620.06 & 101.66 & $\cdots$ & $\cdots$ & $8.76(0.02)$ & $6.94(0.02)$ & $5.49(0.01)$ & $\ldots$ & $\ldots$ & $\cdots$ & $\cdots$ & $\cdots$ & & $\mathrm{ABU}$ \\
\hline 2013 & $\begin{array}{l}\text { NOV } \\
24.04\end{array}$ & 6620.54 & 102.14 & $\cdots$ & $\cdots$ & $\cdots$ & $\cdots$ & $5.2(0.15)$ & $3.3(0.38)$ & $2.0(0.25)$ & $0.9(0.35)$ & $-0.2(0.4)$ & $-0.2(0.2)$ & & OBO \\
\hline
\end{tabular}




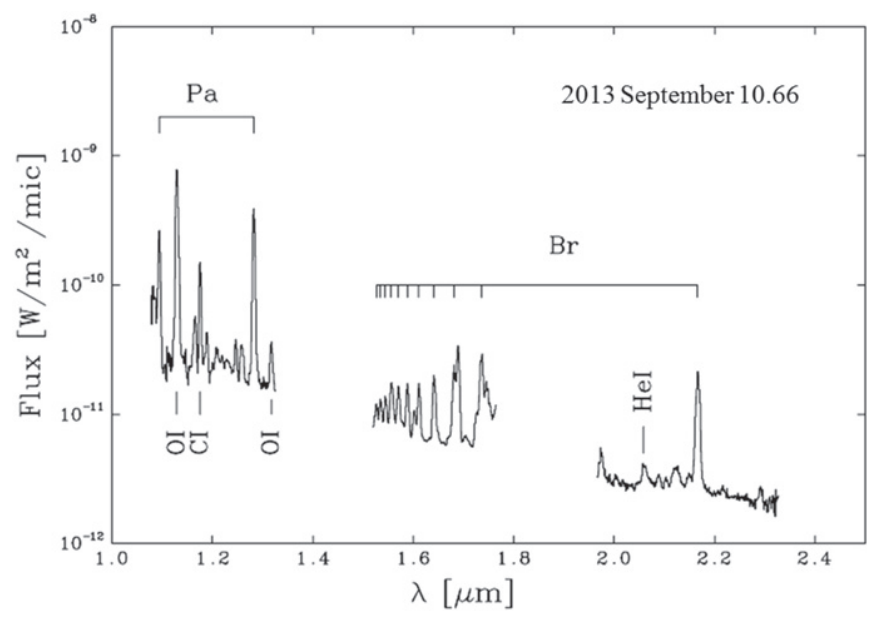

Figure 3. $1.08-2.35 \mu \mathrm{m}$ Mt. Abu spectrum obtained on 2013 September 10.66 (JD 2456546.16, day 27.76). The spectrum establishes the nova to be of the Fe II class, spectral templates of which are given in Banerjee \& Ashok (2012). Prominent lines seen are those of the Paschen and Brackett series of hydrogen, $\mathrm{O}_{\mathrm{I}} 1.1287$ (Ly $\beta$ fluoresced) and $\mathrm{O}_{\mathrm{I}} 1.3164$, $\mathrm{He}_{\mathrm{I}} 1.0830$ and $2.0581 \mu \mathrm{m}$. Notable are the several $\mathrm{C}_{\mathrm{I}}$ lines seen which are hallmarks in the NIR to separate the $\mathrm{Fe}$ II from the $\mathrm{He} / \mathrm{N}$ class of novae. Some of the strongest $\mathrm{C}_{\mathrm{I}}$ lines are seen at $1.165,1.175,1.188$, and $1.689 \mu \mathrm{m}$ (Das et al. 2008).

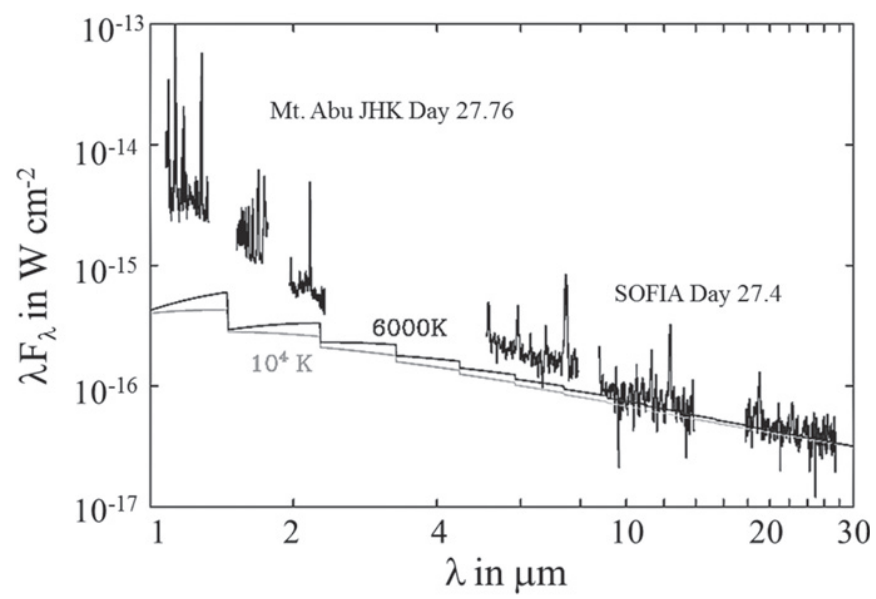

Figure 4. Combined Mt. Abu and SOFIA Day 27.4 spectrum from 1.04 to $28 \mu \mathrm{m}$. The slope of the spectrum beyond $5 \mu \mathrm{m}$ is $\lambda F_{\lambda} \propto \lambda^{-1}$ as would be expected for optically thin free-free radiation. No metallic forbidden lines appear in the entire spectrum. The curves are the expected contribution from free-free and bound-free radiation; see text for details.

times the Eddington limit for a one solar mass WD. A super Eddington luminosity $\mathrm{CN}$ eruption on a $\mathrm{CO}$ WD is not unprecedented. Schwarz et al. (2001) reported that the CO Nova LMC 1991 reached a maximum luminosity of $6 \times 10^{5}$ $L_{\odot}(\sim 10$ times the Eddington Luminosity for a one solar mass WD) before visual maximum. An alternate method of estimating the outburst luminosity is to use the maximum magnitude rate of decline relationships (MMRDs) derived by Downes \& Duerbeck (2000). These give the peak absolute visual magnitude, $M_{\mathrm{v}}$, as a function of the times, $t_{2}$ and $t_{3}$, taken for the visual light to decline from maximum by two and three magnitudes respectively. From the photometric visual light recorded by Munari et al. (2013a) we find $t_{2} \sim 11.0^{\mathrm{d}}$ and $t_{3} \sim 20.0^{\mathrm{d}}$. The Downes and Duerbeck MMRDs yield a value of $M_{\mathrm{v}} \sim-8.7$ and, assuming a bolometric correction of approximately zero, $M_{\text {bol }} \sim-8.7$ and $L_{\mathrm{o}} \sim 2.6 \times 10^{5} L_{\odot}$. This is further confirmation that V339 Del was super-Eddington near maximum light.

\section{DISCUSSION}

\subsection{The Ejected Gas Mass}

The mass of the ionized gas ejected can be estimated using the free-free emission model described by Ennis et al. (1977), Gehrz et al. (1980a), Gehrz (1999, 2008), and Evans \& Gehrz (2012). This method uses the relationship between the electron density, $n_{\mathrm{e}}$, and the cut-off wavelength, $\lambda_{\mathrm{c}}$, at which the shell becomes optically thick. When $\lambda_{c}$ can be determined observationally as shown in Figure 6(b), Gehrz (1999, 2008) and Evans \& Gehrz (2012) have shown that the ejected gas mass can be determined by:

$$
M_{\text {gas }}=1.5 \times 10^{-12} f\left(V_{\mathrm{o}} t\right)^{5 / 2} \lambda_{c}^{-1} \text { in } M_{\odot}
$$

where $\lambda_{\mathrm{c}}$ is the cut-off wavelength in $\mu \mathrm{m}, V_{\mathrm{o}}$ is the expansion velocity in $\mathrm{km} \mathrm{s}^{-1}, \mathrm{t}$ is the time elapsed since the outburst, and $f$ is the ratio of the thickness to the radius of the ejected shell. As described in Section 3 above, our expansion velocities are derived from IR hydrogen lines that are good tracers of the gas kinematics because they arise from the bulk of the ejecta. Emission lines from hydrogen, whether they arise in the optical or the IR can be assumed to arise from the same regions and hence will be co-spatial and have the same kinematics (expansion velocity). We find for V339 Del that $\lambda_{\mathrm{c}} \sim 2.2 \mu \mathrm{m}$ on day 3.25 , which was the only date upon which an inflection was seen. As was the case for Nova Her 1991 (Woodward et al. 1992), this inflection quickly moved out of the observable window of our photometers and had moved beyond $5 \mu \mathrm{m}$ by the next observation on Day 7.27. If the time dependence of the electron density is assumed to be $n_{\mathrm{e}} \propto t^{-q}$, then for $\lambda_{\mathrm{c}}$ to have moved beyond $5 \mu \mathrm{m}$ by day $7.27 q$ would be $\geqslant 1$ for a shell expanding at constant thickness and $q$ would be $\geqslant 1.55$ for a shell in free expansion. Hauschildt et al. (1992) have found through modeling that the density in nova photospheres goes as $n_{\mathrm{e}} \propto t^{-2}-t^{-3}$, and we assume that $n_{\mathrm{e}} \propto t^{-2}$ in the discussion that follows. Following the arguments put forth by Skopal et al. (2014), we assume that $f \sim 1$ as would be expected for a freely expanding shell with a unity filling factor. Adopting a value for $V_{\mathrm{o}}$ of $505 \mathrm{~km} \mathrm{~s}^{-1}$ for the expansion velocity, the ejected gas mass is $\sim 7.5 \times 10^{-5} M_{\odot}$. Given the spherically symmetric shell geometry with a filling factor of unity used to derive Equation (5), the electron density associated with the shell on day 3.25 would be $n_{\mathrm{e}} \sim 10^{13} \mathrm{~cm}^{-3}$. We note that our result is consistent with the findings of Skopal et al. (2014), who obtained a value for the ionized ejecta of $\sim 9.3 \times 10^{-5} M_{\odot}$ on 2013 August 28 (Day 14) and "a few times $10^{-4} M_{\odot}$ " by the end of the mass ejection phase.

An alternative estimate of the ejected hydrogen mass can be obtained by using the HI recombination lines, provided that they are optically thin. To determine if this assumption is valid, we examined the line ratios in the SOFIA spectrum. The ratios 

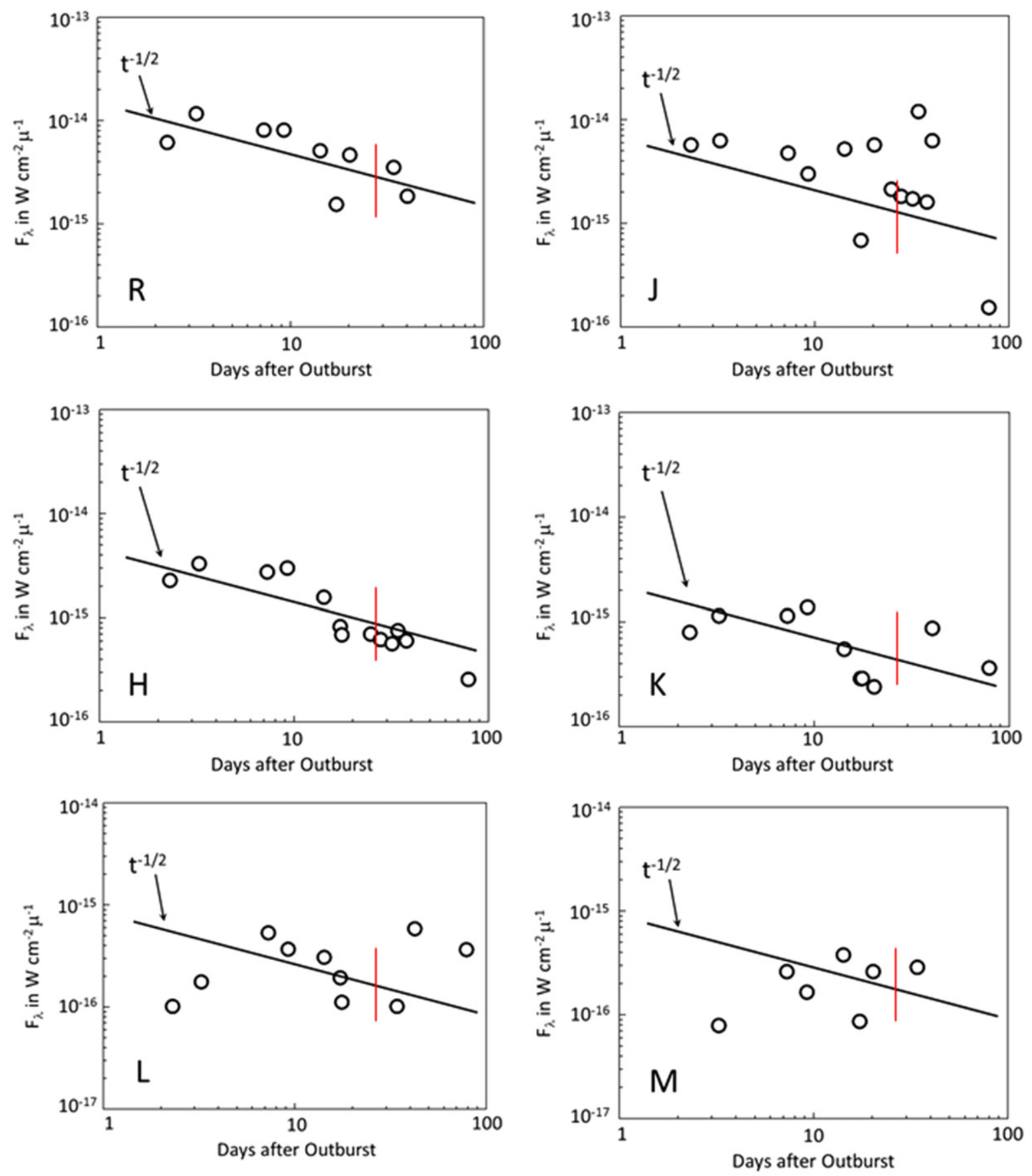

Figure 5. RJHKLM light curves during the first 100 days for V339 Del from the photometry summarized in Table 4. The early near-IR light decline trends roughly as $t^{-1 / 2}$ (with large excursions due to changing emission line strengths) as was the case for the $2.3 \mu \mathrm{m}$ light of the dust forming CO nova LW Ser (Gehrz et al. 1980a) and the near-IR light curves of PW Vul (Gehrz et al. 1988). The rising $K, L$, and $M$ light after $\sim 40-50$ days probably due to dust formation. The Vertical red lines mark the time at which the SOFIA spectrum was obtained.

are provided in Table 3 . We then compared these values to predictions based on the Case B assumption as calculated by Storey \& Hummer (1995). We were unable to reproduce the observed line ratios for any reasonable combination of temperature $\left(5000 \mathrm{~K}<=T_{\mathrm{e}}<=15000 \mathrm{~K}\right)$ or density $\left(10^{5} \mathrm{~cm}^{-3} \leqslant n_{\mathrm{e}} \leqslant 10^{14} \mathrm{~cm}^{-3}\right)$. A similar exercise for the NIR data was done by comparing the relative strengths of the several Brackett lines seen in the $H$ and $K$ bands with Case B predictions. Here too we find the Brackett lines to be optically thick (see Figure 8). This means that the assumptions of Case B are invalid at this stage of development, i.e., the Lyman series ( $u \rightarrow 2$, where $u$ is the principal quantum number of the upper level) optical depth is so high that thermal collisional excitation and de-excitation dominates radiative de-excitation. Consequently, ejecta mass estimates based on the hydrogen emission lines will be lower limits since the lines are quenched. Even though the emission lines are optically thick, we were still able to use them to calculate a lower limit to the ejecta mass.

We use the HI 8-7 $(19.062 \mu \mathrm{m}), 11-9(22.340 \mu \mathrm{m}), 9-7$ $(11.309 \mu \mathrm{m}), \quad 12-8 \quad(10.504 \mu \mathrm{m}), \quad 12-7 \quad(6.772 \mu \mathrm{m}), \quad 13-7$ 

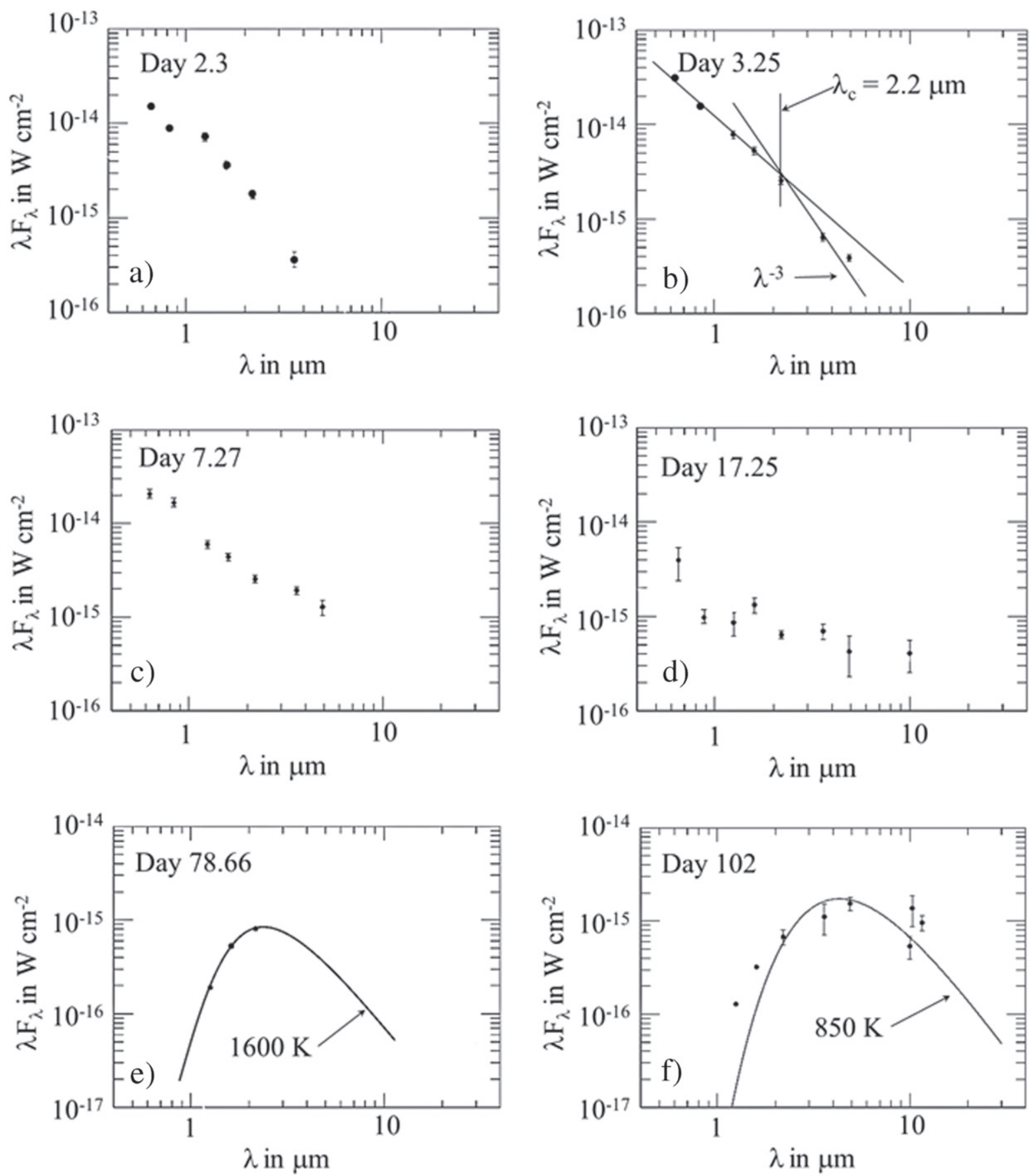

Figure 6. Spectral energy distributions (SEDs) of V339 Del at several critical epochs. Except where indicated, the $1 \sigma$ error bars are smaller than the plotted data points unless otherwise indicated. Data have been de-reddened assuming $E(B-V)=0.18$. Panel (a) shows the Day 2.3 OBO SED that is typical of the fireball expansion phase. Panel (b): the Day 3.25 OBO SED shows the cut-off wavelength, $\lambda_{\mathrm{c}}=2.2 \mu \mathrm{m}$, where the SED turns over onto the Rayleigh-Jeans tail of a blackbody. Panels (c) and (d) show OBO SEDs typical of free-free with superimposed hydrogen recombination lines. It is evident that the density has evolved to that $\lambda_{\mathrm{c}}$ has moved to longer wavelengths. Panel (e): Mt. Abu data showing that weak dust emission was present on day 78.66. The unusually high temperature suggests that the grains had just begun to nucleate and were very small. Panel (f) OBO SED shows that the dust emission had increased significantly by Day 102 .

$(6.292 \mu \mathrm{m})$ and $10-6(5.129 \mu \mathrm{m})$ lines which are unblended with other lines (e.g., the $8-6$ line at $7.503 \mu \mathrm{m}$ is contaminated by the 11-7 line at $7.506 \mu \mathrm{m})$. For the optically thin case, the flux $f\left(\mathrm{~W} \mathrm{~m}^{-2}\right)$ in the line $u-\ell$ is

$$
f=N_{u} A_{u l} \frac{h c}{\lambda_{u l}} \frac{V}{4 \pi D^{2}}
$$

where $A_{u}$ is the Einstein coefficient, $N_{u}$ is the number of $\mathrm{H}$ atoms per unit volume in the upper level and $V$ is the volume of the gas. Thus,

$$
f=b_{u} \frac{M_{\mathrm{H}}}{m_{\mathrm{H}}} n_{\mathrm{e}} \frac{h^{3}}{\left[2 \pi m_{\mathrm{e}} k T_{\mathrm{e}}\right]^{3 / 2}} u^{2} e^{h R / u^{2} k T_{\mathrm{e}}} \frac{A_{u}}{\lambda_{u}} \frac{h c}{4 \pi D^{2}}
$$

where $M_{\mathrm{H}}$ is the total $\mathrm{H}$ mass, $m_{\mathrm{H}}$ is the mass of a $\mathrm{H}$ atom, $b_{u}$ is the factor that describes the departure from thermal equilibrium, and $n_{e}\left(\mathrm{~cm}^{-3}\right)$ is the electron density. The values of $b_{u}$ are taken from Storey \& Hummer (1995); these depend on 

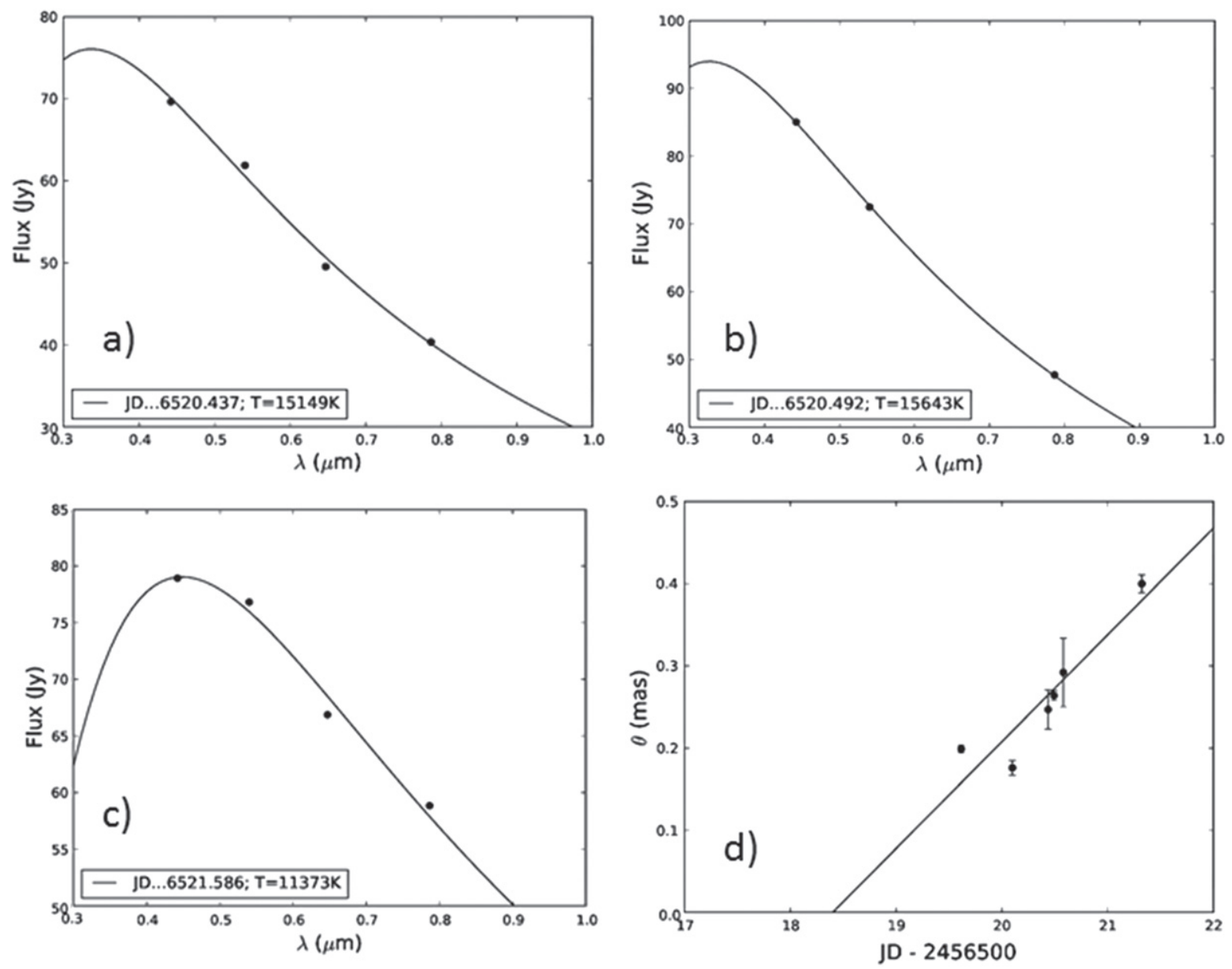

Figure 7. Panels (a)-(c) show the development of the blackbody temperature of the fireball and panel (d) shows the fit that gives the expansion rate. Data have been de-reddened assuming $E(B-V)=0.18$. The error bars are $1 \sigma$.

electron temperature and density, and on the value of $u$. They are typically between 0.7 and 1 . The other quantities have their usual meanings. Solving for $M_{\mathrm{H}} / M_{\odot}$, we have:

$\frac{M_{\mathrm{H}}}{M_{\odot}}=1.222 \times 10^{7} \frac{D_{\mathrm{kpc}}^{2}}{n_{\mathrm{e}}\left(\mathrm{cm}^{-3}\right)} \frac{1}{b_{u} u^{2}} \frac{\lambda_{u}}{A_{u}} e^{-\frac{15.774}{u^{2}}} f\left(10^{-16} \mathrm{Wm}^{-2}\right)$

for a temperature of $10^{4} \mathrm{~K}$. Assuming that the electron density evolves as $n_{\mathrm{e}} \propto t^{-2}$ as discussed earlier, it will have declined to $n_{\mathrm{e}} \sim 10^{11} \mathrm{~cm}^{-3}$ by day 27.1 given the value of $n_{\mathrm{e}} \sim 10^{13} \mathrm{~cm}^{-3}$ inferred from the day 3.25 free-free cut-off wavelength. At this high density, it is not surprising that the SOFIA FORCAST mid-IR spectrum is devoid of detectable forbidden line emission. The IR forbidden lines in this spectral range are well quenched at densities greater than $n_{\mathrm{e}} \sim 10^{6}-10^{7} \mathrm{~cm}^{-3}$. Equation (8) yields a lower limit to the ejected hydrogen mass of $M_{\text {gas }} \sim 5.0 \times 10^{-6} M_{\odot}$. This result is significantly lower than that estimated from the day 3.25 free-free cut-off wavelength, but is not surprising since the emission lines are optically thick. Further it is possible that the electron density scales as $t^{-3}$ as would be expected from a ballistically ejected constant mass shell, in which case the use of Equation (8) will give an improved and increased mass estimate closer to $10^{-5}$ $M_{\odot}$. A ballistic, or Hubble flow, for the ejecta's velocity has been found to apply to several novae (e.g., Shore et al. 2013a and references therein). In Figure 4 we have included the free-free and bound-free continuum for a gas of ionized hydrogen with $n_{\mathrm{e}} \sim 1.4 \times 10^{11} \mathrm{~cm}^{-3}$ at $6000 \mathrm{~K}$ and $10^{4} \mathrm{~K}$ (the contribution of ionized helium is negligible in these conditions); the continuum is normalized to the SOFIA data at $10 \mu \mathrm{m}$. It is evident that the NIR continuum falls well below the Mt Abu data at $1-2.5 \mu \mathrm{m}$, indicating that there is another source of radiation at the shorter wavelengths. We presently have no plausible explanation for this NIR excess. An elementary semi-quantitative argument shows that the excess is unlikely to be due to an accretion disk. The absolute bolometric magnitude of a $\mathrm{CN}$ system that is dominated by an accretion disk is $\sim+4.2$, and the system has a high enough color temperature so that its $V /$ IR color is zero (Prialnik \& Kovetz 1992). At a distance of $4.5 \mathrm{kpc}$, the apparent NIR magnitude of an accretion disk would therefore be $\sim 17.5$. On day 27 the nova had an apparent $J H K$ magnitudes of $\sim 5.5$, i.e., 12 mag brighter than would be expected for an accretion disk.

\subsection{Dust Formation and the Mass of the Dust}

A very small amount of dust formed in the ejecta of V339 Del as evidenced by our OBO photometry during 2013 November 19-24. Our IR photometry on 2013 November 24.04 (JD 2456620.54, day 102.14) is consistent with the 
Table 5

Data from the Fireball Expansion Phase

\begin{tabular}{lccccc}
\hline \hline No. & Filters & JD & Fireball Blackbody Temperature $(\mathrm{K})$ & $\left(\lambda f_{\lambda}\right)_{\max }\left(10^{-14} \mathrm{~W} \mathrm{~cm}^{-2}\right)$ & Fireball Angular Diameter $(\mathrm{mas})$ \\
\hline 1 & $B V R I$ & 6519.615 & $13,612 \pm 149$ & $3.32 \pm .084$ & $0.199 \pm 0.005$ \\
2 & $B V R I$ & 6520.099 & $17,164 \pm 405$ & $6.59 \pm 0.313$ & $0.176 \pm 0.009$ \\
3 & $B V R I$ & 6520.437 & $15,149 \pm 659$ & $7.85 \pm 0.739$ & $0.247 \pm 0.024$ \\
4 & $B V I$ & 6520.492 & $15,643 \pm 148$ & $10.21 \pm 0.21$ & $0.264 \pm 0.006$ \\
5 & $B V R I$ & 6520.581 & $14,690 \pm 932$ & $9.74 \pm 1.362$ & $0.292 \pm 0.042$ \\
6 & $B V I$ & 6521.323 & $11,861 \pm 140$ & $7.77 \pm 0.238$ & $0.400 \pm 0.011$ \\
\hline
\end{tabular}

Table 6

Expansion Velocities of the Principal Ejecta from the 2013 September 10.66 Mt. Abu Spectrum At $R=\Delta \lambda / \lambda \sim 1000$

\begin{tabular}{|c|c|c|c|c|c|c|c|}
\hline$\overline{\lambda_{\mathrm{o}}(\mu \mathrm{m})}$ & $\Delta \lambda(\mu \mathrm{m})$ & Line ID & Peak flux $\left(\mathrm{W} \mathrm{m}^{-2} \mu \mathrm{m}^{-1}\right)$ & \pm & Line flux $\left(\mathrm{W} \mathrm{m}^{-2}\right)$ & \pm & Velocity $\left(\mathrm{Km} \mathrm{s}^{-1}\right)$ \\
\hline 2.1655 & $8.51 \times 10^{-3}$ & $\mathrm{Br} 7$ & $1.75 \times 10^{-11}$ & $2.80 \times 10^{-13}$ & $1.58 \times 10^{-13}$ & $2.54 \times 10^{-15}$ & 571 \\
\hline 1.6407 & $6.14 \times 10^{-3}$ & Br12 & $1.32 \times 10^{-11}$ & $2.33 \times 10^{-13}$ & $8.60 \times 10^{-14}$ & $1.52 \times 10^{-15}$ & 542 \\
\hline 1.2818 & $4.96 \times 10^{-3}$ & $\mathrm{~Pa} 5$ & $3.67 \times 10^{-10}$ & $3.39 \times 10^{-12}$ & $1.94 \times 10^{-12}$ & $1.79 \times 10^{-14}$ & 561 \\
\hline 1.5342 & $4.15 \times 10^{-3}$ & $\operatorname{Br} 18$ & $3.93 \times 10^{-12}$ & $2.27 \times 10^{-13}$ & $1.74 \times 10^{-14}$ & $1.02 \times 10^{-15}$ & 379 \\
\hline 1.5439 & $4.68 \times 10^{-3}$ & Br17 & $4.92 \times 10^{-12}$ & $2.09 \times 10^{-13}$ & $2.45 \times 10^{-14}$ & $1.04 \times 10^{-15}$ & 431 \\
\hline 1.5556 & $5.95 \times 10^{-3}$ & Br16 & $8.98 \times 10^{-12}$ & $1.90 \times 10^{-13}$ & $5.68 \times 10^{-14}$ & $1.21 \times 10^{-15}$ & 554 \\
\hline 1.5700 & $5.61 \times 10^{-3}$ & Br15 & $7.81 \times 10^{-12}$ & $1.99 \times 10^{-13}$ & $4.67 \times 10^{-14}$ & $1.19 \times 10^{-15}$ & 512 \\
\hline 1.5881 & $5.35 \times 10^{-3}$ & Br14 & $9.55 \times 10^{-12}$ & $2.01 \times 10^{-13}$ & $5.44 \times 10^{-14}$ & $1.15 \times 10^{-15}$ & 484 \\
\hline 1.6109 & $5.69 \times 10^{-3}$ & Br13 & $1.03 \times 10^{-11}$ & $1.99 \times 10^{-13}$ & $6.21 \times 10^{-14}$ & $1.24 \times 10^{-15}$ & 510 \\
\hline
\end{tabular}

presence of a dust shell having a blackbody temperature of $T \sim$ $850 \mathrm{~K} \pm 40$ and a flux maximum of $\left(\lambda F_{\lambda}\right)_{\max } \sim$ $2.0 \times 10^{-15} \mathrm{~W} \mathrm{~cm}^{-2}$. This type of SED is consistent with the assumption that the type of dust formed was primarily amorphous carbon. The total luminosity of the dust shell was only $\sim 0.02 L_{\mathrm{o}}$, suggesting that the amount of dust formed was quite low. Assuming as is the case for other dust forming novae, that the grains are sub-micron size with an emissivity $Q_{\mathrm{e}}$ $\propto a T^{2}$, where a is the grain radius and $T$ is the grain temperature, Gehrz et al. (1980a, 1980b), Gehrz (1999, 2008), and Evans \& Gehrz (2012) have shown that the dust mass is given by:

$$
M_{\mathrm{dust}}=4.81 \times 10^{23} \frac{\rho_{\mathrm{d}} D^{2}\left(\lambda F_{\lambda}\right)_{\max }}{T_{\mathrm{BB}}^{6}} \text { in } M_{\odot}
$$

where $D$ is in kpc and $\rho_{\mathrm{d}}$ is the dust grain density in $\mathrm{gm} \mathrm{cm}^{-3}$. In the case of V339 Del, for amorphous carbon grains with $\rho_{\mathrm{d}}$ $\sim 2.25 \mathrm{gm} \mathrm{cm}^{-3}$ (see e.g., Iati et al. 2001), Equation (9) yields $M_{\mathrm{d}} \sim(1.2 \pm 0.4) \times 10^{-7} M_{\odot}$. Taranova et al. (2014) have independently concluded from JHKLM photometry obtained during the first sixty days after outburst that V339 Del had produced only $\sim 4.5 \times 10^{-9} M_{\odot}$ of dust by 2013 October 11 (JD 2456576.5, day 58.1). Our JHK Mt. Abu photometry on Day 78.66, if interpreted as due to warm dust at a temperature of $\sim 1600 \mathrm{~K}$, would imply a dust mass of $\sim 1.3 \times 10^{-9} M_{\odot}$. Although this grain temperature is higher than previously seen in nova carbon dust, Sellgren (1984) has argued that stochastic heating of very small grains can produce high temperatures. An alternative explanation for the high color temperature is that emission lines may still be present in the $H$ and $K$ bands. The large increase in the dust mass between days 58 and 102 is consistent with the rapid rate of grain size growth that occurs following grain nucleation in dust forming novae (see Shore \& Gehrz 2004).
The failure of this nova to produce substantial quantities of dust can be understood in terms of the criterion derived by Gehrz \& Ney (1987) that characterizes the critical density, $\rho_{\text {crit }}$, when the ejected material reaches the blackbody distance for $1000 \mathrm{~K}$ grains for optically thick dust shell formation in $\mathrm{CNe}$ :

$$
\begin{aligned}
\rho \geqslant \rho_{\text {crit }} & =1.2 \times 10^{-4} \frac{\left(M_{\mathrm{gas}} / M_{\odot}\right)}{\left(L_{\mathrm{o}} / L_{\odot}\right)^{3 / 2}} \mathrm{gm} \mathrm{cm}^{-3} \\
& =3 \times 10^{-16} \mathrm{gm} \mathrm{cm}^{-3} .
\end{aligned}
$$

In Equation (10), $M_{\text {gas }}$ is the mass of the gas ejected and $L_{\mathrm{o}}$ is the outburst luminosity. In the case of V339 Del, Equation (10) yields a value of $1.2 \times 10^{-17} \mathrm{gm} \mathrm{cm}^{-3}$ at the blackbody distance, far below the mean density associated with novae that produce significant amounts of dust. The very high ejected hydrogen gas mass to carbon dust mass ratio of $M_{\text {gas }} / M_{\text {dust }} \sim$ 470-940 is consistent with inefficient dust production in V339 Del. Complete condensation of the carbon in a gas with solar abundance would produce $M_{\text {gas }} / M_{\text {dust }} \sim 177$, and CO novae are expected to produce large overabundances of carbon (Gehrz et al. 1998).

The dust formation in this nova also showed a peculiarity that has perhaps not been exhibited by any other nova: the dust persisted and co-existed in the presence of a soft X-ray radiation field that is expected to have destroyed it. Also the early gamma radiation (Ackermann et al. 2014) might have inhibited the formation of nucleation sites. The destruction of dust due to irradiation by $\mathrm{X}$ - and gamma-radiation has been discussed in detail by Fruchter et al. (2001). Observationally, the dust commenced formation around the end of September (Shenavrin et al. 2013) while the super-soft X-ray phase began and intensified by the end of September and the beginning of 2013 October (Page \& Beardmore 2013; Page et al. 2013). A possible interpretation of this behavior is that the dust was protected by residing in regions into which the X-rays could 


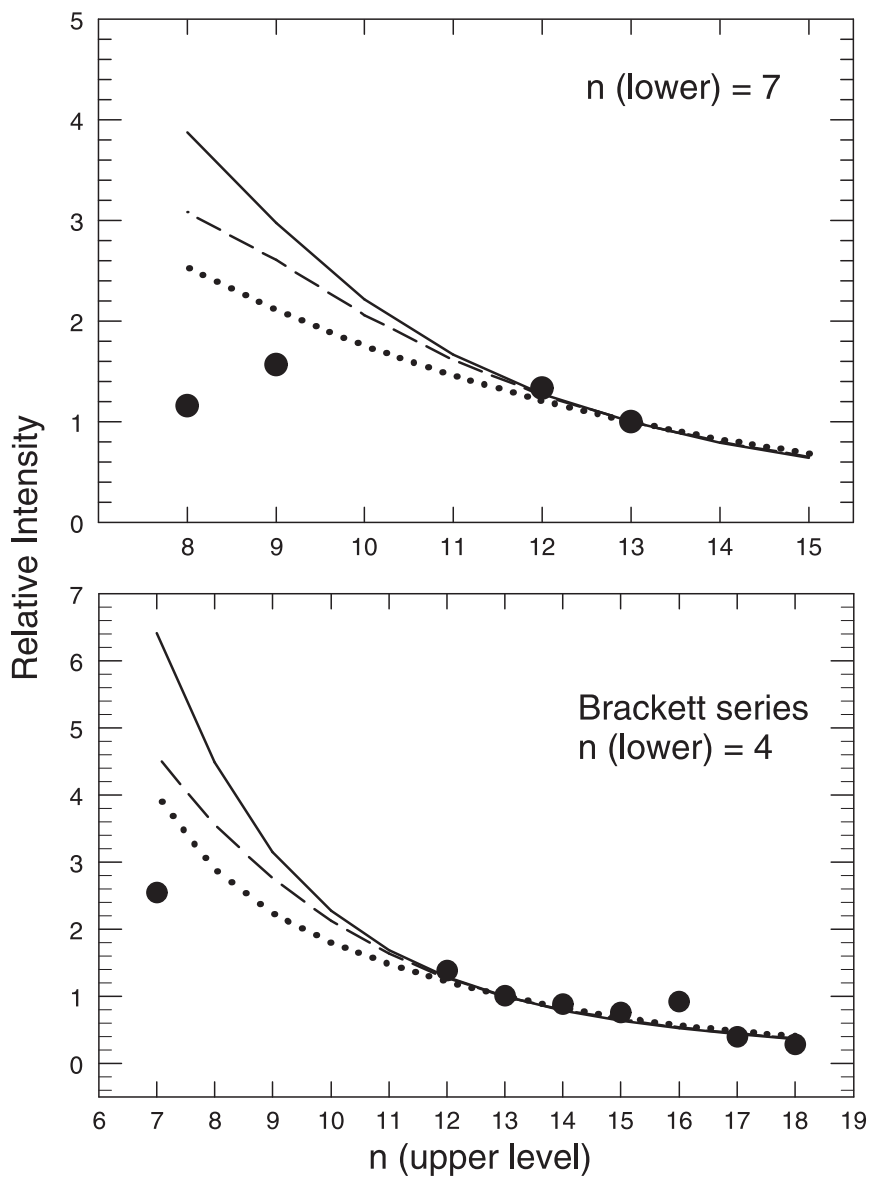

Figure 8. Representative comparison of observed line strengths (black filled circles) with Case B predictions. Upper panel is for SOFIA data for transitions ending in a lower level $n=7$ with $n=13-7$ normalized to unity. The lower panel is for the NIR Brackett lines with $n=13-4$ normalized to unity. The Case B curves are for $T=10,000 \mathrm{~K}$ and representative values of the electron density $=10^{9}, 10^{10}$ and $10^{11} \mathrm{~cm}^{-3}$ indicated by the dotted, dashed and continuous lines respectively. The deviation seen between the observed values and Case B values, indicative of optical depth effects, persists for any other reasonable combination of the electron density and temperature.

not fully penetrate, e.g., dense clumps or a thick equatorial density enhancement. Invoking an equatorial overdensity for the system is attractive for another reason since such overdensities are known to collimate outflowing ejecta into a hourglass or bipolar shape (Ribeiro et al. 2014) — a suggestion of such a shape was seen in the interferometric studies of V339 Del by Schaefer et al. (2014).

\section{CONCLUSIONS}

We have presented ground-based IR photometry and $J H K$ spectroscopy and 5-28 $\mu \mathrm{m}$ SOFIA FORCAST spectroscopy documenting the early temporal development of Nova Delphini 2013 (V339 Del). The $J H K$ spectroscopy establishes the nova to be of the Fe II class. The unique composite $1-28 \mu \mathrm{m}$ spectrum presented here can thus serve as an useful template for comparison of spectral features of other Fe II type novae observed in the future in this near to mid-IR range.

We derive a distance of $\sim 4.5 \mathrm{kpc}$ from data available from the early expansion of the fireball. This distance gives an outburst luminosity of $\sim 8.3 \times 10^{5} L_{\odot}$ making V339 Del the most luminous CO nova on record. Our data provide new constraints on the ejected gas mass and the dust yield in fast
CO novae. The ejected gas mass is estimated by several methods to be $\sim 10^{-4} M_{\odot}$. There is evidence for the formation of $\sim 1.2( \pm 0.4) \times 10^{-7} M_{\odot}$ of dust about 50 days after outburst. The gas to dust ratio of $\sim 470 / 1-940 / 1$ implies that dust production was much less efficient in V339 Del than is the case for most $\mathrm{CO}$ novae.

We are grateful to an anonymous referee for suggestions that substantially improved our presentation and interpretation of the data. We thank the British Astronomical Association/The Astronomer magazine for the visual light curve data. R. D. G., C. E. W., and L. A. H. were supported by a USRA SOFIA Cycle 1 Target of Opportunity Nova contract. R. D. G. received support from the United States Air Force. T. L. acknowledges the support of the Estonian Ministry for Education and Science. The research at the Physical Research Laboratory is supported by the Department of Space, Government of India. S. S. was supported by NSF and NASA grants to ASU. The OBO observations were supported, in part, by a generous gift from Edward Glickman.

\section{REFERENCES}

Ackermann, M., Ajello, M., Albert, A., Baldini, L., et al. 2014, Sci, 345, 554 Banerjee, D. P. K., \& Ashok, N. M. 2012, BASI, 40, 243

Banerjee, D. P. K., Ashok, N. M., Joshi, V., \& Evans, A. 2013a, ATel, \#5337 Banerjee, D. P. K., Ashok, N. M., Joshi, V., \& Evans, A. 2013b, ATel, \#5404 Cass, A., Carlon, R. L., Corgan, D. T., et al. 2013, ATel, \#5434 Clarke, M., Vacca, W. D., \& Shuping, R. Y. 2015, in ASP Conf. Ser., ADASS XXIV, ed. A. R. Taylor \& J. M. Stil (San Francisco, CA: ASP), 495, 355 Cushing, M. C., Vacca, W. D., \& Rayner, J. T. 2004, PASP, 116, 362

Das, R. K., Banerjee, D. P. K., Ashok, N. M., \& Chesneau, O. 2008, MNRAS, 391, 1874

Downes, R. A., \& Duerbeck, H. W. 2000, AJ, 120, 2007

Edwards, L. O. V., Fadda, D., Biviano, A., \& Marleau, F. R. 2010, AJ, 139,434

Ennis, D., Becklin, E. E., Beckwith, S., et al. 1977, ApJ, 214, 478

Evans, A., Callus, C. M., Whitelock, P. A., \& Laney, D. 1990, MNRAS, 246, 527

Evans, A., \& Gehrz, R. D. 2012, BASI, 40, 213

Evans, A., \& Rawlings, J. M. C. 2008, in Classical Novae, ed. M. F. Bode \& A. Evans (Cambridge: Cambridge Univ. Press), 308

Fruchter, A., Krolik, J. H., \& Rhoads, J. E. 2001, ApJ, 563, 597

Gallagher, J. S., \& Ney, E. P. 1976, ApJL, 204, L35

Gehrz, R. D. 1988, ARA\&A, 26, 377

Gehrz, R. D. 1999, PhR, 311, 405

Gehrz, R. D. 1997, ICQ, 19, 55

Gehrz, R. D. 2008, in Classical Novae, ed. M. F. Bode \& A. Evans (Cambridge: Cambridge Univ. Press), 167

Gehrz, R. D., Evans, A., \& Woodward, C. E. 2014, in Proc. ASP Conf. Ser. 490, "Stella Novae: Future and Past Decades", ed. P. A. Woudt \& V. A. R. M. Ribeiro (San Francisco, CA: ASP), 227

Gehrz, R. D., Grasdalen, G. L., \& Hackwell, J. A. 1985, ApJL, 298, L47 (erratum 1986, ApJL, 306, L49)

Gehrz, R. D., Grasdalen, G. L., Hackwell, J. A., \& Ney, E. P. 1980a, ApJ, 237, 855

Gehrz, R. D., Hackwell, J. A., Grasdalen, G. L., et al. 1980b, ApJ, 239, 570

Gehrz, R. D., Harrison, T. E., Ney, E. P., et al. 1988, ApJ, 329, 894

Gehrz, R. D., \& Ney, E. P. 1987, PNAS (USA), 84, 6961

Gehrz, R. D., \& Ney, E. P. 1992, Icar, 100, 162

Gehrz, R. D., Truran, J. W., Williams, R. E., \& Starrfield, S. 1998, PASP, 110,3

Hauschildt, P. H., Wehrse, R., Starrfield, S., \& Shaviv, G. 1992, ApJ, 393, 307 Helton, L. A., Gehrz, R. D., Woodward, C. E., et al. 2012, ApJ, 755, 37

Herter, T., Adams, J. D., De Buizer, J. M., et al. 2012, ApJL, 749, L18

Herter, T. L., Vacca, W. D., Adams, J. D., Keller, L. D., et al. 2013, PASP, 125,1393

Iati, M. A., Cecchi-Pestellini, C., Williams, D. A., et al. 2001, MNRAS, 322, 749

Kolotilov, E. A. 1980, SvAL, 6, 268

Munari, U., Henden, A., Dallaporta, S., \& Cherini, G. 2013a, IBVS, 6080

Munari, U., Valisa, P., Milani, A., \& Cetrulo, G. 2013b, ATel, 5297 
Nakano, S., Itagaki, K., Denisenko, D., et al. 2013, CBET, 3628

Page, K. L., \& Beardmore, A. P. 2013, ATel, \#5429

Page, K. L., Osborne, J. P., Kuin, N. P. M., et al. 2013, ATel, \#5470

Prabhu, T. P., \& Anupama, G. C. 1987a, Ap\&SS, 131, 479

Prabhu, T. P., \& Anupama, G. C. 1987b, JApA, 8, 369

Prialnik, D., \& Kovetz, A. 1992, ApJ, 385, 665

Rayner, J. T., Toomey, D. W., Onaka, P. M., et al. 2003, PASP, 115, 362

Ribeiro, V. A. R. M. 2014, in Proc. ASP Conf. Ser. 490, "Stella Novae: Future and Past Decades", ed. P. A. Woudt \& V. A. R. M. Ribeiro (San Francisco, CA: ASP), 129

Ribeiro, V. A. R. M., Chomiuk, L., Munari, U., et al. 2014, ApJ, 792, 57

Rieke, G. H., \& Lebofsky, M. J. 1985, ApJ, 288, 618

Schaefer, G. H., Brummelaar, T. T., Gies, D. R., et al. 2014, Natur, 515, 234

Schwarz, G. J., Shore, S. N., Starrfield, S., et al. 2001, MNRAS, 320, 103
Sellgren, K. 1984, ApJ, 277, 623

Shenavrin, V. I., Taranova, O. G., \& Tatarnikov, A. M. 2013, ATel, \#5431

Shore, S. N., \& Gehrz, R. D. 2004, A\&A, 417, 695

Shore, S. N., Schwarz, G. J., De Gennaro, A. I., et al. 2013a, A\&A, 549, A140

Shore, S. N., Skoda, P., Korakova, D., et al. 2013b, ATel, 5312

Skopal, A., Dreschel, H., Tarasova, T., et al. 2014, A\&A, 569, 112

Storey, P. J., \& Hummer, D. G. 1995, MNRAS, 272, 41

Taranova, O. G., Tatarnikov, A. M., Shenavrin, V. I., \& Tatarnikova, A. A. 2014, AstL, 40, 120

Temi, P., Marcum, P. M., Young, E., et al. 2014, ApJS, 212, 24

Woodward, C. E., Gehrz, R. D., Jones, T. J., \& Lawrence, G. F. 1992, ApJ, 384, 41

Young, E. T., Becklin, E. E., Marcum, P. M., et al. 2012, ApJL, 749, L17 